\title{
Estimation of zonal errors in star catalogs using CCD observations of ICRF sources
}

\author{
A. Fienga ${ }^{1}$ and A. H. Andrei ${ }^{2}$ \\ ${ }^{1}$ Institut de mécanique céleste et de calcul des éphémérides - Observatoire de Paris, EP 1825 CNRS, \\ 77 avenue Denfert-Rochereau, 75014 Paris, France \\ ${ }^{2}$ Grupo de Estudos em Astronomia-OV/UFRJ and Observatório Nacional/MCT, R. Gal. José Cristino 77, S. Cristovão, \\ R. Janeiro, Brasil
}

Received 10 April 2002 / Accepted 2 July 2002

\begin{abstract}
ICRF sources were observed and positions were deduced using USNO A2.0 stars and stars extracted from the preliminary version of the GSC II catalog, named GSC2.2. These positions were compared to the ICRF positions, and to other published positions of the same optical counterparts. A significant distortion of the USNO A2.0 catalog is revealed for stars fainter than the 11th magnitude and with declinations from 0 to $20 \mathrm{deg}$. On the other hand, the preliminary version of the GSC II catalog shows a very good external accuracy and a good homogeneity in right ascension and declination for the observed zones.
\end{abstract}

Key words. astrometry - catalogs

\section{Introduction}

Since 1997 and following Resolution B2 of the XXIIIrd IAU General Assembly (IAU 1997), the ICRF (Ma et al. 1998) is the official reference frame of the International Astronomical Union. This reference frame is materialized by a catalog of about 600 extragalactic radio sources (Arias 1995) observed by VLBI (Johnston et al. 1995). Because of the great distance between the ground based observers and the source of emission of these objects, they do not have apparent proper motions on the milliarcsecond per year level. Thus, they are the best representation of a non-rotating reference frame at the accuracy of the VLBI observations. Furthermore, thanks to the high accuracy of the VLBI astrometry, the ICRF represents a major improving in the accuracy of the fundamental astrometric reference frame (Feissel \& Mignard 1998). However, an optical representation of such reference frame is needed for optical observations. The Hipparcos catalog (Kovalevsky et al. 1997) was chosen for the optical materialization of the ICRF. Since 1997, numerous stellar catalogs (Tycho, ACT, USNO A2.0) have been linked to the ICRF reference frame via the Hipparcos catalog (ESA 1997; Urban et al. 1998; Monet et al. 1998). However, the accuracy of such links as well as the external uncertainties of the catalogs need to be investigated.

One way to study the external accuracy of the stellar catalogs is to compute positions of the ICRF sources using star positions extracted from the catalog we want to study. One can then compare the computed positions with the official ICRF

Send offprint requests to: A. Fienga, e-mail: fienga@bdl.fr positions and the positions deduced from other catalogs. By this method, possible zonal or regional defects of the catalog as well as mis-linkages with the ICRF can be detected.

In 2001, the first preliminary version of the new GSC II catalog, named GSC 2.2 was published (STScI 2001). This preliminary version is dense and presents improved accuracies in astrometry and photometry. The total number of available objects in April 2002 is 455851237 (526280881 in the USNO A2.0) with a limit magnitude of 18.5 in F and 19.5 in J. The estimated accuracy of the preliminary version of GSC II is about 0.3 arcsec.

In this work, we present, in Sect. 1, the observations of 38 optical counterparts of ICRF sources. In Sect. 2, we describe the applied reduction process based on the use of USNO A2.0 and GSC 2.2 stars. In Sect. 3, we give the results and the comparison with ICRF positions and other published positions of optical counterparts. We discuss the results of such comparisons and conclude with a possible detection of zonal errors of the USNO A2.0 catalog and a first estimation of the external accuracy of the preliminary version of the GSC II catalog compared to USNO A2.0 catalog.

\section{Optical observations}

The observations were made at Observatoire de HauteProvence, France, using the $1.20 \mathrm{~m}$ telescope. The focal length is about $7 \mathrm{~m}$. The CCD has 1024 by 1024 pixels with a scale of 0.68 arcsec per pixel. The field is about 144 squared arcmin. Each source was observed three times with the Cousins $V$ and $R$ filters (540 and $610 \mathrm{~nm}$ ). The observation campaign took 

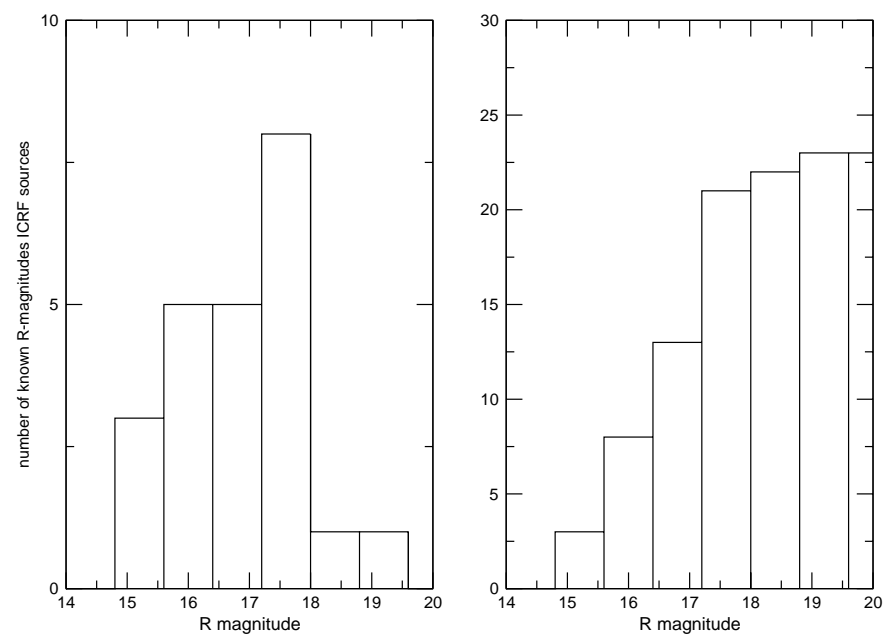

Fig. 1. Histograms and cumulative histograms of the $R$ magnitudes of the 23 optical counterparts. Only $23 R$ magnitudes were published for the 38 optical counterparts studied in this work.
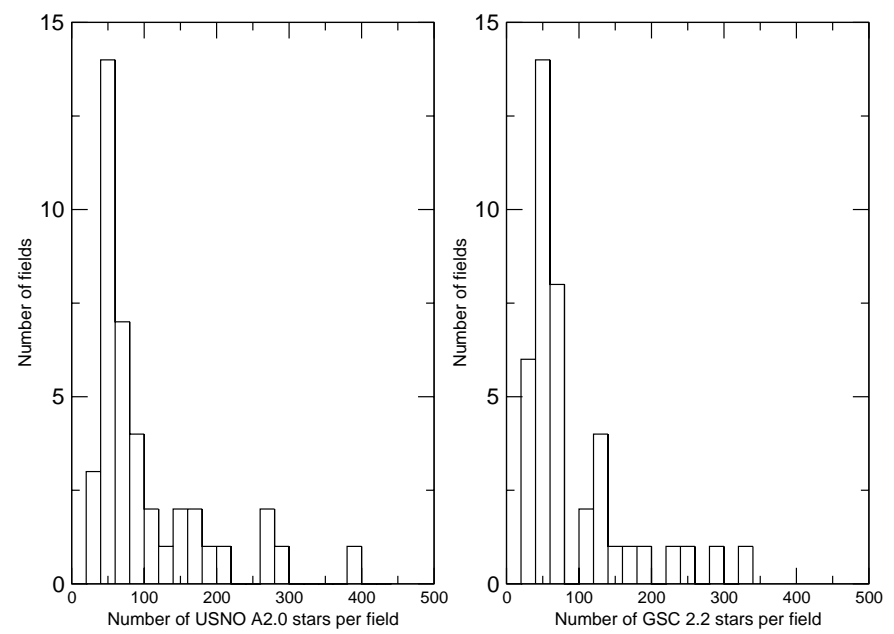

Fig. 2. Number of USNO A2.0 stars and GSC 2.2 stars used for the reduction of each optical counterparts.

place from 2001 December the third to December the tenth. The latitude of the Observatoire de Haute-Provence allows the observation of the full northern portion of the ICRF, where $73 \%$ of its defining sources are located. For the present run, the targets selection was made to accommodate tying sources with the southern observation program (Vieira Martins 2001).

The flatfielding and the bias corrections were made using the CCD reduction package ECLIPSE 4.2 (2002). The extraction of the sources was realized with an automatic extraction procedure (Alard 2002). For the astrometric reduction process, the software PRIAM was used (Fienga 1999; Fienga et al. 2002). The input of PRIAM consisted of the extracted source coordinates in pixels, the star catalog coordinates and a series of observational characteristics such as the geodetic coordinates of the observation site, the meteorological parameters of observation, and the wavelengths of observation. The reduction is done including computation of apparent places (corrections of annual and diurnal aberrations, deflection of light), corrections of refraction by mapping functions dealing with the
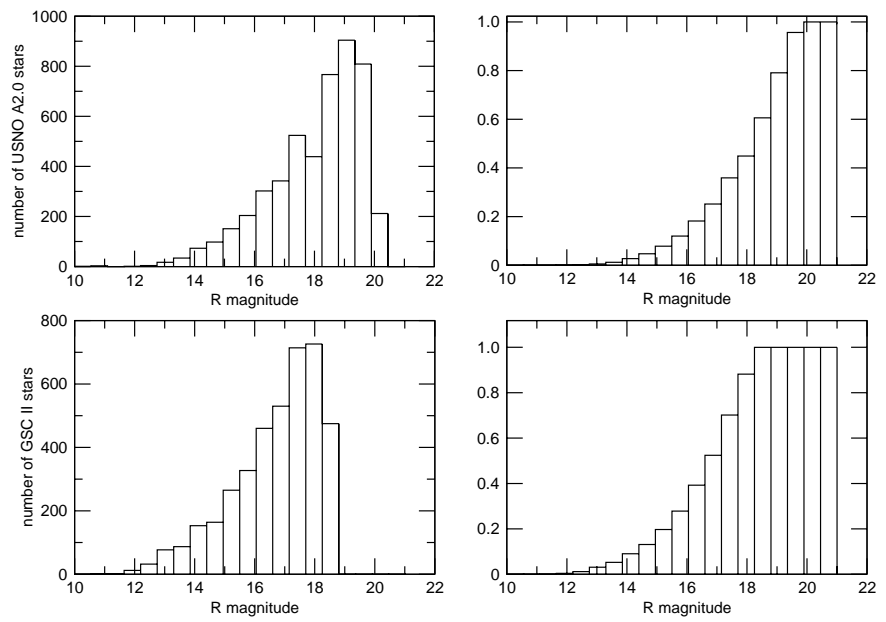

Fig. 3. Histograms and cumulative histograms of the $R$ magnitudes of USNO A2.0 and GSC 2.2 stars used for the astrometric reduction of the optical counterparts.

meteorological conditions of observations and the wavelengths of observations. An easy-to-use interface was built on the web (www.bdl.fr/priam.html). The objects are observed from 23 hours to 13 hours in right ascension and from 0 to $45 \mathrm{deg}$ in declination. The visual magnitudes of the sources are fainter than the 11th magnitude as well as the magnitude of the stars used in the reduction process (see Figs. 1 and 3). For the reduction process, we choose to use the USNO A2.0 catalog and the preliminary version of the GSC II catalog. The observed positions deduced from such reductions would be positions of optical counterparts of ICRF sources in USNO A2.0 and GSC II frames. The offsets between these USNO and GSC2.2 referred positions and the radio source coordinates in the ICRF, Hipparcos or Tycho reference frames would give an idea of how accurate the links between these stellar catalogs are. In Table 1, the visual magnitudes and the ICRF positions of the observed sources are given as well as the number of USNO A2.0 and GSC 2.2 stars used in the reduction process. Column 3 gives the references of the other optical published positions. 6 optical counterparts were observed for the first time. Figure 2 shows the distributions of the number of USNO A2.0 and GSC 2.2 stars used for each field in the reduction process. The average number of stars used in the reduction is about 60 USNO A2.0 and GSC 2.2 stars. Figure 3 shows histograms and cumulative histograms of the distributions of the $R$-magnitudes of USNO A2.0 and GSC 2.2 stars used in the reduction. Thanks to the cumulative histograms, one can see that $85 \%$ of the USNO A2.0 stars and $70 \%$ of the GSC 2.2 stars used for the determination of the positions of the optical counterparts have a brightness fainter than the 16th magnitude.

\section{Results and discussion}

\subsection{Reference catalogs}

Two catalogs were selected for the reduction process.

The USNO A2.0 catalog (Monet et al. 1998), contains 526280881 objects, including positions for 452 ICRF sources. This catalog was made from a high precision astrometric and 

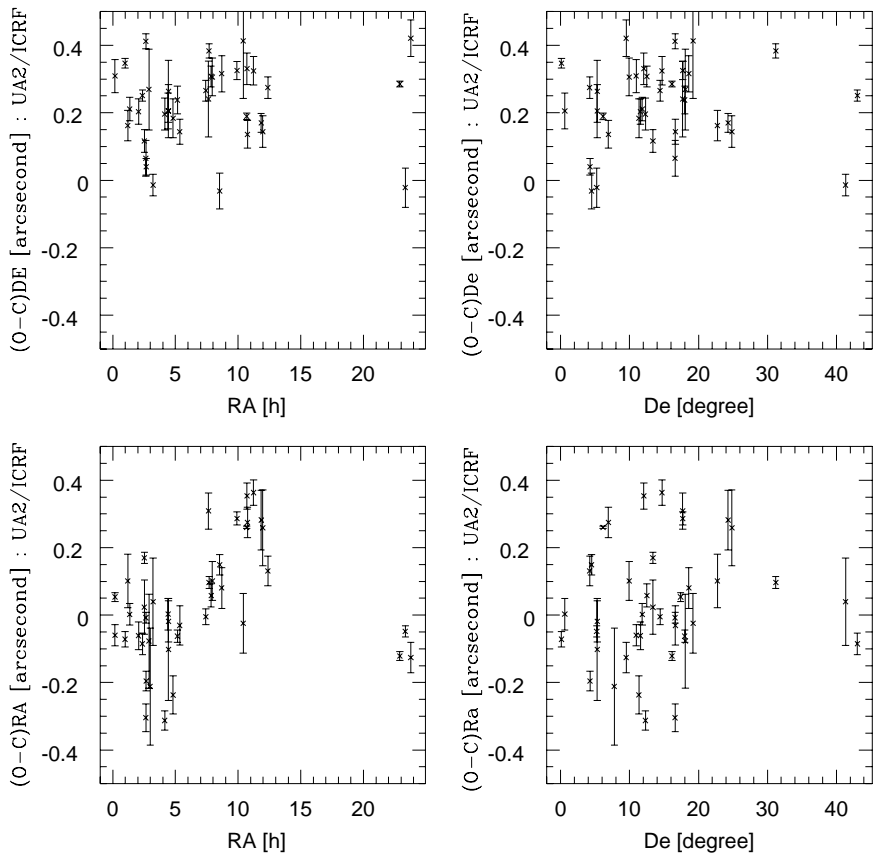

Fig. 4. Normal points of observed positions of ICRF optical counterparts computed with USNO A2.0 stars. The error bars are the standard errors of the observed positions minus the ICRF positions.

photometric reduction of plates from the POSS, SERC and ESO surveys. The positions are referred to the ACT, at the plates epoch. The standard deviation for the USNO A2.0 optical minus radio differences of the ICRF sources is 217 mas, matching well the catalog formal errors.

In 2001, the first preliminary version of the new GSC II catalog, named GSC 2.2 was published (STScI 2001). This preliminary version presents improved accuracies in astrometry and photometry. The total number of available objects in April 2002 is 455851237 with a limit magnitude of 18.5 in F and 19.5 in J. The estimated accuracy of the preliminary version of GSC II is about 0.3 arcsec.

The USNO A2.0 and the GSC 2.2 catalogs do not include proper motions, parallaxes and radial velocities. This means that the motion of the reference stars from the epoch of the plates used to set up the catalogs to the date of observation of the ICRF counterparts cannot be taken into account. As the difference in epochs can be as large as 48 years, the positional effects for a given star could be also large. But, since the reference stars usually are quite distant, especially the faint ones, the net effect will mostly be to increase the random noise on the determination of the ICRF source optical counterpart positions. There are however two sources of systematic errors from proper motion components. The presumably largest one is due to the reciprocal of the solar motion towards the apex, and it cannot be taken into account for lack of parallaxes for the catalogs stars. The other is due to the galactic rotation, and is independent of distance. They both are much smaller than the main source of errors in the reduction, which comes from the uncertainties in the catalog position for the reference stars.
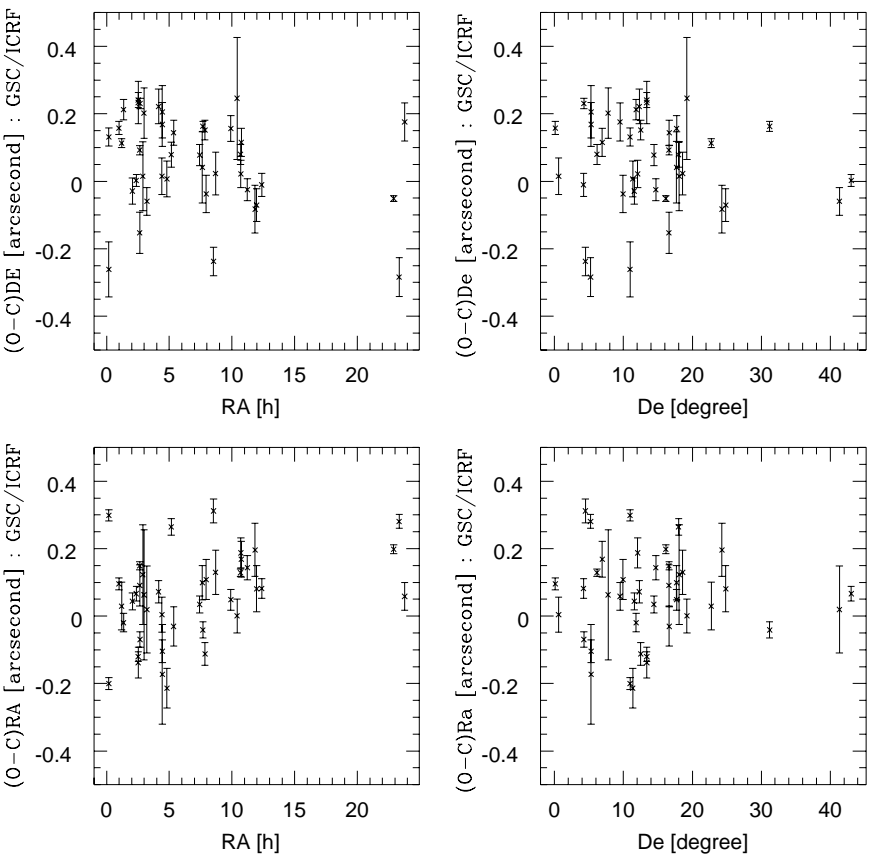

Fig. 5. Normal points of observed positions of ICRF optical counterparts computed with GSC 2.2 stars. The error bars are the the standard errors of the observed positions minus the ICRF positions.

\subsection{USNO and GSC positions compared to ICRF positions}

Figures 4 and 5 show the differences between the observed positions deduced from USNO A2.0 stars and GSC 2.2 stars and the ICRF positions. Theoretically, such residuals should not represent only the offsets between the stellar catalogs. They also contain offsets between the positions of the radio sources and their optical counterparts. The structure observed in the radio images of the ICRF sources (Charlot 1990; Fey \& Charlot 1997) degrade the radio positions on the sub-milliarcsecond level. The differencein position induced by the structure of the source will not appear in our residuals because of the lower precision and accuracy of the optical observations of the counterparts (Zacharias et al. 1999). In Fig. 4 there is a clear offset in declination of the USNO A2.0 residuals. This offset in declination $(254 \pm 153)$ mas does not appear in GSC residuals $(59 \pm 138)$ mas, meaning that it is induced by the star catalogs used in the reduction process. Figure 6 shows the distribution of the residuals versus the published magnitudes of the ICRF optical counterparts. No variations of the residuals seem to be connected with the magnitudes of the counterparts. This indicates that magnitude-dependent systematic errors are not significant in our residuals. Furthermore, Assafin et al. (2001) detected offsets in the USNO A2.0 star declinations by comparison between the catalogs USNO A2.0, ACT (Urban et al. 1998) and the improved GSC (Morrison et al. 1999). A sharp discontinuity was detected in the USNO A2.0 catalog at declination $-20 \mathrm{deg}$ as well as some indication of a zonal error around declination $+30 \mathrm{deg}$ for stars fainter than the 11 th magnitude. The plate measurements used to set up the USNO catalog were referred to the ACT, meaning that stars fainter than the ACT magnitude range could be affected by zonal 
Table 1. List of observed ICRS sources. Columns 1, 4 and 5 give the official ICRS names and positions of the observed sources. The visual magnitude of each source is given in Col. 2 when known. In Col. 3, 1 indicates that the source was observed by Zacharias et al. (1999), 2 indicates that the source was observed by Da Silva Neto et al. (2000) and b indicates that the source was observed by both. Columns 5 and 6 give the numbers of USNO A2.0 and GSC 2.2 stars used in the reduction of each field. The last four columns give the residuals in right ascension and declination computed by comparison between the ICRF positions and the observed positions computed with the USNO A2.0 and GSC 2.2 catalogs.

\begin{tabular}{|c|c|c|c|c|c|c|c|c|c|c|}
\hline \multirow{2}{*}{$\begin{array}{l}\text { ICRF } \\
\text { Source }\end{array}$} & \multirow[t]{2}{*}{$m$} & & \multirow{2}{*}{$\begin{array}{l}\text { ICRF RA } \\
\text { h m s.sssss }\end{array}$} & \multirow{2}{*}{$\begin{array}{l}\text { ICRF Dec } \\
\text { d m s.ssss }\end{array}$} & \multirow[t]{2}{*}{ UA2 } & \multirow[t]{2}{*}{ GSC } & \multicolumn{2}{|c|}{ UA2 -ICRF } & \multicolumn{2}{|c|}{ GSC 2.2 -ICRF } \\
\hline & & & & & & & $\begin{array}{r}\Delta \alpha \cos \delta \\
{[\mathrm{mas}]}\end{array}$ & $\begin{array}{r}\Delta \delta \\
{[\mathrm{mas}]}\end{array}$ & $\begin{array}{r}\Delta \alpha \cos \delta \\
{[\mathrm{mas}]}\end{array}$ & $\begin{array}{r}\Delta \delta \\
{[\mathrm{mas}]}\end{array}$ \\
\hline $0007+106$ & 15.4 & $\mathrm{~b}$ & 001031.00588 & +105829.5041 & 50 & 80 & $169_{ \pm 167}$ & $116_{ \pm 34}$ & $299_{ \pm 16}$ & $132_{ \pm 27}$ \\
\hline $0007+171$ & 18.0 & $\mathrm{~b}$ & 001033.99061 & +172418.7613 & 70 & 50 & $-60_{ \pm 32}$ & $309_{ \pm 49}$ & $-200_{ \pm 18}$ & $-262_{ \pm 81}$ \\
\hline 0056-001 & 17.7 & 1 & 005905.51464 & +000651.6221 & 45 & 36 & $-72_{ \pm 23}$ & $347_{ \pm 14}$ & $96_{ \pm 18}$ & $158_{ \pm 19}$ \\
\hline $0109+224$ & 15.7 & $\mathrm{~b}$ & 011205.52471 & +224438.7862 & 35 & 30 & $101_{ \pm 79}$ & $162_{ \pm 45}$ & $30_{ \pm 71}$ & $113_{ \pm 13}$ \\
\hline $0119+115$ & & & 012141.59504 & +114950.4131 & 77 & 62 & $2_{ \pm 32}$ & $211_{ \pm 34}$ & $-20_{ \pm 28}$ & $212_{ \pm 30}$ \\
\hline $0201+113$ & & & 020340.65706 & +113445.4096 & 47 & 45 & $-61_{ \pm 41}$ & $203_{ \pm 38}$ & $44_{ \pm 25}$ & $-29_{ \pm 39}$ \\
\hline $0219+428$ & & 2 & 022239.61150 & +430207.7988 & 160 & 150 & $-85_{ \pm 32}$ & $251_{ \pm 17}$ & $66_{ \pm 22}$ & $3_{ \pm 17}$ \\
\hline $0229+131$ & 17.7 & 1 & 023145.89405 & +132254.7163 & 60 & 45 & $38_{ \pm 81}$ & $588_{ \pm 55}$ & $-129_{ \pm 46}$ & $237_{ \pm 62}$ \\
\hline $0235+164$ & 15.5 & b & 023838.93011 & +163659.2747 & 53 & 52 & $-156_{ \pm 40}$ & $239_{ \pm 53}$ & $149_{ \pm 13}$ & $92_{ \pm 14}$ \\
\hline $0237+040$ & 18.5 & 1 & 023951.26305 & +041621.4188 & 68 & 46 & $-196_{ \pm 29}$ & $40_{ \pm 24}$ & $-69_{ \pm 23}$ & $231_{ \pm 15}$ \\
\hline $0250+178$ & & & 025334.88230 & +180542.5238 & 70 & 70 & $-77_{ \pm 139}$ & $269_{ \pm 120}$ & $123_{ \pm 148}$ & $15_{ \pm 102}$ \\
\hline $0256+075$ & 18.0 & $\mathrm{~b}$ & 025927.07663 & +074739.6432 & 43 & 45 & $211_{ \pm 173}$ & $587_{ \pm 85}$ & $63_{ \pm 193}$ & $202_{ \pm 75}$ \\
\hline $0309+411$ & & 2 & 031301.96213 & +412001.1835 & 270 & 250 & $40_{ \pm 130}$ & $-14_{ \pm 32}$ & $20_{ \pm 129}$ & $-60_{ \pm 41}$ \\
\hline $0406+121$ & & 2 & 040922.00874 & +121739.8475 & 60 & 60 & $-313_{ \pm 28}$ & $196_{ \pm 47}$ & $72_{ \pm 33}$ & $222_{ \pm 51}$ \\
\hline $0422+004$ & 17.0 & $\mathrm{~b}$ & 042446.84205 & +003606.3298 & 90 & 70 & $3_{ \pm 47}$ & $205_{ \pm 53}$ & $4_{ \pm 52}$ & $15_{ \pm 54}$ \\
\hline $0423+051$ & 19.5 & b & 042636.60410 & +051819.8720 & 80 & 66 & $-18_{ \pm 61}$ & $205_{ \pm 78}$ & $-104_{ \pm 34}$ & $168_{ \pm 65}$ \\
\hline $0446+112$ & & 2 & 044907.67120 & +112128.5966 & 143 & 124 & $-237_{ \pm 56}$ & $184_{ \pm 58}$ & $-214_{ \pm 58}$ & $7_{ \pm 53}$ \\
\hline $0507+179$ & & 2 & 051002.36912 & +180041.5817 & 300 & 330 & $-62_{ \pm 18}$ & $238_{ \pm 41}$ & $265_{ \pm 25}$ & $79_{ \pm 37}$ \\
\hline $0518+165$ & & 2 & 052109.88602 & +163822.0512 & 400 & 300 & $-31_{ \pm 58}$ & $143_{ \pm 37}$ & $-31_{ \pm 58}$ & $144_{ \pm 37}$ \\
\hline $0722+145$ & & 2 & 072516.80775 & +142513.7468 & 280 & 230 & $-5_{ \pm 23}$ & $266_{ \pm 31}$ & $35_{ \pm 25}$ & $77_{ \pm 31}$ \\
\hline $0735+178$ & 16.2 & 1 & 073807.39374 & +174218.9983 & 170 & 130 & $309_{ \pm 53}$ & $241_{ \pm 112}$ & $99_{ \pm 51}$ & $41_{ \pm 105}$ \\
\hline $0738+313$ & 16.1 & $\mathrm{~b}$ & 074110.70331 & +311200.2286 & 180 & 140 & $97_{ \pm 17}$ & $384_{ \pm 21}$ & $-41_{ \pm 24}$ & $163_{ \pm 15}$ \\
\hline $0748+126$ & 18.0 & 1 & 075052.04573 & +123104.8282 & 210 & 190 & $58_{ \pm 34}$ & $308_{ \pm 31}$ & $-112_{ \pm 34}$ & $152_{ \pm 29}$ \\
\hline $0754+100$ & 15.0 & b & 075706.64294 & +095634.8521 & 200 & 170 & $101_{ \pm 58}$ & $306_{ \pm 56}$ & $108_{ \pm 59}$ & $-38_{ \pm 55}$ \\
\hline $0829+046$ & 16.4 & $\mathrm{~b}$ & 083148.87695 & +042939.0853 & 140 & 130 & $150_{ \pm 31}$ & $-32_{ \pm 53}$ & $312_{ \pm 36}$ & $-238_{ \pm 42}$ \\
\hline $0839+187$ & 16.4 & $\mathrm{~b}$ & 084205.09418 & +183540.9906 & 90 & 80 & $80_{ \pm 61}$ & $316_{ \pm 54}$ & $130_{ \pm 66}$ & $23_{ \pm 63}$ \\
\hline $0952+179$ & 17.2 & $\mathrm{~b}$ & 095456.82363 & +174331.2224 & 45 & 32 & $286_{ \pm 19}$ & $325_{ \pm 27}$ & $48_{ \pm 31}$ & $157_{ \pm 38}$ \\
\hline $1022+194$ & 17.5 & 1 & 102444.80959 & +191220.4153 & 30 & 25 & $-24_{ \pm 88}$ & $413_{ \pm 171}$ & $0_{ \pm 50}$ & $246_{ \pm 181}$ \\
\hline $1038+064$ & 16.7 & $\mathrm{~b}$ & 104117.16250 & +061016.9238 & 65 & 45 & $260_{ \pm 3}$ & $189_{ \pm 9}$ & $129_{ \pm 11}$ & $80_{ \pm 30}$ \\
\hline $1040+123$ & 17.3 & $\mathrm{~b}$ & 104244.60521 & +120331.2641 & 53 & 36 & $353_{ \pm 39}$ & $331_{ \pm 47}$ & $187_{ \pm 45}$ & $22_{ \pm 40}$ \\
\hline $1042+071$ & & & 104455.91126 & +065538.2625 & 60 & 50 & $275_{ \pm 45}$ & $136_{ \pm 41}$ & $169_{ \pm 53}$ & $115_{ \pm 42}$ \\
\hline $1111+149$ & 18.0 & $\mathrm{~b}$ & 111358.69510 & +144226.9526 & 58 & 58 & $363_{ \pm 38}$ & $324_{ \pm 42}$ & $144_{ \pm 36}$ & $-25_{ \pm 32}$ \\
\hline $1147+245$ & & 2 & 115019.21217 & +241753.8350 & 26 & 25 & $282_{ \pm 88}$ & $170_{ \pm 28}$ & $196_{ \pm 79}$ & $-83_{ \pm 71}$ \\
\hline $1155+251$ & & & 115825.78750 & +245017.9637 & 44 & 48 & $259_{ \pm 112}$ & $144_{ \pm 47}$ & $81_{ \pm 68}$ & $-70_{ \pm 48}$ \\
\hline $1219+044$ & & 2 & 122222.54962 & +041315.7763 & 70 & 50 & $131_{ \pm 43}$ & $275_{ \pm 32}$ & $82_{ \pm 29}$ & $-11_{ \pm 34}$ \\
\hline $2251+158$ & 16.1 & 1 & 225357.74794 & +160853.5609 & 115 & 105 & $-121_{ \pm 13}$ & $285_{ \pm 9}$ & $198_{ \pm 13}$ & $-51_{ \pm 8}$ \\
\hline $2318+049$ & & & 232044.85660 & +051349.9527 & 95 & 73 & $-48_{ \pm 16}$ & $-22_{ \pm 58}$ & $281_{ \pm 21}$ & $-284_{ \pm 58}$ \\
\hline $2344+092$ & 16.0 & 1 & 234636.83856 & +093045.5149 & 83 & 55 & $-126_{ \pm 45}$ & $421_{ \pm 54}$ & $58_{ \pm 41}$ & $176_{ \pm 56}$ \\
\hline
\end{tabular}

errors. In Fig. 3, the cumulative histogram of the USNO A2.0 stars magnitude distribution shows that more than $99 \%$ of the stars used in the reduction process are fainter than the 11th magnitude. Such a zonal error of the USNOA2.0 catalog described by Assafin et al. (2001) could affect the observed positions of the ICRF optical counterparts. In Table 2, we compute the residuals for 4 declination bins. The 2 last bins are not significant because of the small numbers of sources in these 

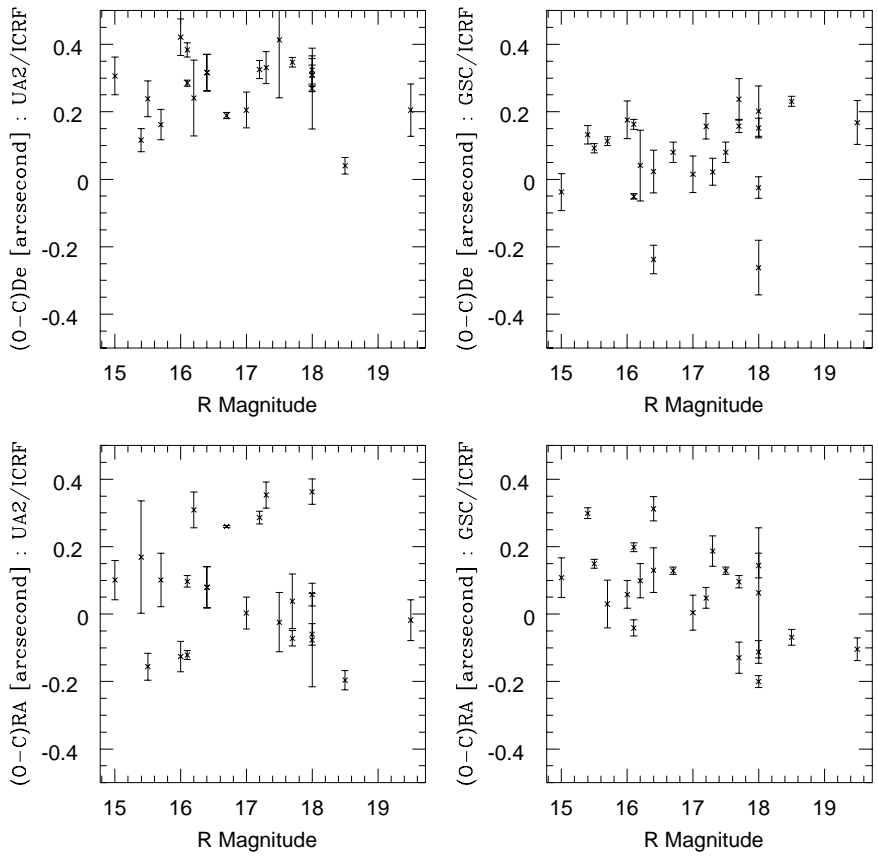

Fig. 6. Normal points of observed positions of ICRF optical counterparts computed with USNO A2.0 and GSC 2.2 stars versus published $R$-magnitudes of optical counterparts. The error bars are the the standard errors of the observed positions minus the ICRF positions.

zones. However, the two first bins present significant offsets in declination for USNO A2.0 ( $225 \pm 175$ mas from 0 to $10 \mathrm{deg}$ and $291 \pm 139$ mas from 10 to $20 \mathrm{deg}$ ) and no significant offset for GSC $(60 \pm 166$ mas from 0 to $10 \mathrm{deg}$ and $71 \pm 131$ mas from 10 to $20 \mathrm{deg})$.

\subsection{USNO and GSC positions compared to other published optical positions}

To compare the observed positions with other positions of optical counterparts, we used the values published by Da Silva Neto et al. (2000) and Zacharias et al. (1999).

Da Silva Neto et al. (2000) reduced 315 optical counterparts of ICRF sources from the DSS plates using secondary reference stars tied to the ACT, Hipparcos and Tycho catalogs. Of these, 24 counterparts were studied also in the present work. In Table 3, the residuals (Da Silva Neto et al. 2000) are grouped by zones as presented here. No significant offset can be detected in the residuals between ACT or Hipparcos positions and ICRF positions. By comparison with the residuals obtained with the USNO A2.0 and the GSC 2.2 catalogs for the same 24 sources, we confirm the detection of a zonal error in the USNO A2.0 catalog. This zonal error affects stars fainter than the 11th magnitude and with declinations between 0 and $30 \mathrm{deg}$. In the other hand, the preliminary version of the GSC II catalog shows its very good external accuracy and its good homogeneity in right ascension and declination in the 0 to $45 \mathrm{deg}$ declination zone. Furthermore, thanks to its high density, the dispersions of the residuals are smaller than those obtained with the ACT catalog (see Table 3). In Zacharias et al. (1999), optical positions of ICRF sources were obtained after CCD observations
Table 2. Means and standard deviations, in mas, of the observed minus ICRF right ascension and declination of the 38 ICRF sources observed in optical wavelengths and reduced using the USNO A2.0 catalog (Cols. 3 and 4), and the preliminary version of the GSC II catalog (Cols. 5 and 6).

\begin{tabular}{rrrrrr}
\hline \hline \multirow{2}{*}{$\begin{array}{c}\text { ICRF } \\
\text { Zones }\end{array}$} & \multicolumn{2}{c}{ UA2-ICRF } & \multicolumn{2}{c}{ GSC 2.2-ICRF } \\
& & $\begin{array}{r}\Delta \alpha \cos \delta \\
{[\mathrm{mas}]}\end{array}$ & {$[\mathrm{mas}]$} & $\begin{array}{r}\Delta \alpha \cos \delta \\
{[\mathrm{mas}]}\end{array}$ & $\begin{array}{r}\Delta \delta \\
{[\mathrm{mas}]}\end{array}$ \\
\hline$\left[0^{\circ}, 45^{\circ}\right]$ & 38 & $30_{ \pm 173}$ & $254_{ \pm 153}$ & $57_{ \pm 131}$ & $59_{ \pm 138}$ \\
& & & & & \\
{$\left[0^{\circ}, 10^{\circ}\right]$} & 12 & $11_{ \pm 160}$ & $225_{ \pm 175}$ & $73_{ \pm 139}$ & $60_{ \pm 166}$ \\
{$\left[10^{\circ}, 20^{\circ}\right]$} & 20 & $18_{ \pm 189}$ & $291_{ \pm 139}$ & $47_{ \pm 141}$ & $71_{ \pm 131}$ \\
{$\left[20^{\circ}, 30^{\circ}\right]$} & 3 & $214_{ \pm 98}$ & $158_{ \pm 13}$ & $102_{ \pm 85}$ & $-13_{ \pm 110}$ \\
$30^{\circ}<\delta$ & 3 & $17_{ \pm 93}$ & $207_{ \pm 203}$ & $15_{ \pm 54}$ & $35_{ \pm 115}$ \\
\hline
\end{tabular}

and reduction with Hipparcos linked secondary sets of stars. We found 23 common sources between our observations and the positions published by Zacharias et al. (1999). As in Da Silva Neto et al. (2000), the computation of the positions is done using secondary reference stars linked to Hipparcos cata$\log$. Zacharias et al. adds to the deduced positions kinematic corrections which overcomes the epoch-differences between the secondary reference stars and their links to the Hipparcos stars. In Table 4, the residuals are presented. The dispersions of the Zacharias et al. residuals which are in the Hipparcos system are smaller than those published by Da Silva Neto et al. (2000) because of the high accuracy of the procedure used by Zacharias et al. (densification of the reference stars used in the procedure). The same remarks as in the Da Silva Neto case can be made. The USNO A2.0 still presents an important offset in declination for stars with declination between 0 and $30 \mathrm{deg}$. The preliminary version of the GSC II keeps its good external accuracy despite an offset in declination in the 20 to $30 \mathrm{deg}$ declination zone. This offset is not really significant due to the limited number of sources observed in this region (138 \pm 35 mas for 2 objects).

\subsection{Direct comparisons with Tycho-2 catalog}

Another criterium for the selection of the optical counterparts of ICRF sources observed in our program was the number of the Tycho- 2 stars in the fields. There are 8 Tycho- 2 fields with 5 or more stars matching the CCD area, enabling direct catalog comparisons. Table 5 lists the averaged differences between Tycho-2 positions with GSC 2.2 and USNO A2.0 positions of common stars detected within a radius of 8.5 arcmin centered of the ICRF positions. Theoretically, such comparisons would give another estimation of the possible distortions of the USNO A2.0 catalog and the GSC 2.2 catalog in the Tycho-2 reference frame. However, as the large standard deviations of Table 5 show, the differences in epochs and accuracies between the different catalogs as well as the limited number of common stars in the fields do not allow any significant detection of distortion.

With these results, one can stress again that the astrometric analysis of optical counterparts of ICRF sources is indeed a very powerful tool to investigate warps in stellar catalogs. 
Table 3. Means and standard deviations, in mas, of the observed minus ICRF $\alpha$ and $\delta$ of 24 optical counterparts of ICRF sources The sources were commonly observed by Da Silva Neto et al. and this paper. Column 1 presents the residuals obtained by Da Silva Neto et al. by comparison between their positions obtained with the ACT catalog and ICRF positions. In Col. 2, we have residuals obtained by comparison between Hipparcos positions and ICRF. Our residuals are presented in the last 2 columns.

\begin{tabular}{|c|c|c|c|c|c|c|c|c|}
\hline \multirow[b]{2}{*}{$\delta$ Zones } & \multicolumn{2}{|c|}{$\begin{array}{l}\text { ACT -ICRF } \\
\text { DSN et al. }\end{array}$} & \multicolumn{2}{|c|}{$\begin{array}{l}\text { HIP -ICRF } \\
\text { DSN et al. }\end{array}$} & \multicolumn{2}{|c|}{$\begin{array}{l}\text { UA2 -ICRF } \\
\text { This paper }\end{array}$} & \multicolumn{2}{|c|}{$\begin{array}{c}\text { GSC } 2.2 \text {-ICRF } \\
\text { This paper }\end{array}$} \\
\hline & $\begin{array}{r}\Delta \alpha \cos \delta \\
{[\mathrm{mas}]}\end{array}$ & $\begin{array}{r}\Delta \delta \\
{[\mathrm{mas}]}\end{array}$ & $\begin{array}{r}\Delta \alpha \cos \delta \\
\text { [mas] }\end{array}$ & $\begin{array}{r}\Delta \delta \\
{[\mathrm{mas}]}\end{array}$ & $\begin{array}{r}\Delta \alpha \cos \delta \\
{[\mathrm{mas}]}\end{array}$ & $\begin{array}{r}\Delta \delta \\
{[\mathrm{mas}]}\end{array}$ & $\begin{array}{r}\Delta \alpha \cos \delta \\
{[\mathrm{mas}]}\end{array}$ & $\begin{array}{r}\Delta \delta \\
{[\mathrm{mas}]}\end{array}$ \\
\hline$\left[0^{\circ}, 45^{\circ}\right]$ & $26_{ \pm 190}$ & $-68_{ \pm 157}$ & $-4_{ \pm 157}$ & $-20_{ \pm 168}$ & $58_{ \pm 177}$ & $244_{ \pm 131}$ & $73_{ \pm 134}$ & $49_{ \pm 135}$ \\
\hline$\left[0^{\circ}, 10^{\circ}\right]$ & $-32_{ \pm 144}$ & $-43_{ \pm 88}$ & $-28_{ \pm 147}$ & $33_{ \pm 115}$ & $52_{ \pm 165}$ & $238_{ \pm 200}$ & $81_{ \pm 139}$ & $36_{ \pm 158}$ \\
\hline$\left[10^{\circ}, 20^{\circ}\right]$ & $50_{ \pm 191}$ & $-61_{ \pm 185}$ & $37_{ \pm 146}$ & $-25_{ \pm 199}$ & $49_{ \pm 207}$ & $267_{ \pm 86}$ & $76_{ \pm 154}$ & $63_{ \pm 142}$ \\
\hline$\left[20^{\circ}, 30^{\circ}\right]$ & $-100_{ \pm 92}$ & $-110_{ \pm 173}$ & $-180_{ \pm 187}$ & $-117_{ \pm 176}$ & $160_{ \pm 106}$ & $239_{ \pm 126}$ & $61_{ \pm 122}$ & $64_{ \pm 130}$ \\
\hline $30^{\circ}<\delta$ & $87_{ \pm 338}$ & $-87_{ \pm 150}$ & $40_{ \pm 70}$ & $-3_{ \pm 6}$ & $17_{ \pm 93}$ & $207_{ \pm 203}$ & $15_{ \pm 54}$ & $35_{ \pm 115}$ \\
\hline
\end{tabular}

Table 4. Means and standard deviations, in mas, of the observed minus ICRF $\alpha$ and $\delta$ of 23 optical counterparts of ICRF sources The sources were commonly observed by Zacharias et al. and this paper. Column 1 presents the residuals obtained by Zacharias by comparison between their positions obtained with the Hipparcos catalog and ICRF positions. Our residuals are presented in the last 2 columns.

\begin{tabular}{|c|c|c|c|c|c|c|}
\hline \multirow[b]{2}{*}{$\delta$ Zones } & \multicolumn{2}{|c|}{$\begin{array}{c}\text { HIP-ICRF } \\
\text { Zacharias et al. }\end{array}$} & \multicolumn{2}{|c|}{$\begin{array}{l}\text { UA2-ICRF } \\
\text { This paper }\end{array}$} & \multicolumn{2}{|c|}{$\begin{array}{c}\text { GSC } 2.2 \text {-ICRF } \\
\text { This paper }\end{array}$} \\
\hline & $\begin{array}{r}\Delta \alpha \cos \delta \\
{[\mathrm{mas}]}\end{array}$ & $\begin{array}{r}\Delta \delta \\
{[\mathrm{mas}]}\end{array}$ & $\begin{array}{r}\Delta \alpha \cos \delta \\
{[\mathrm{mas}]}\end{array}$ & $\begin{array}{r}\Delta \delta \\
{[\mathrm{mas}]}\end{array}$ & $\begin{array}{r}\Delta \alpha \cos \delta \\
\text { [mas] }\end{array}$ & $\begin{array}{r}\Delta \delta \\
{[\mathrm{mas}]}\end{array}$ \\
\hline$\left[0^{\circ}, 30^{\circ}\right]$ & $24_{ \pm 53}$ & $22_{ \pm 83}$ & $66_{ \pm 167}$ & $280_{ \pm 134}$ & $60_{ \pm 132}$ & $93_{ \pm 170}$ \\
\hline$\left[0^{\circ}, 10^{\circ}\right]$ & $31_{ \pm 61}$ & $12_{ \pm 47}$ & $-17_{ \pm 156}$ & $252_{ \pm 189}$ & $41_{ \pm 134}$ & $105_{ \pm 144}$ \\
\hline$\left[10^{\circ}, 20^{\circ}\right]$ & $20_{ \pm 53}$ & $40_{ \pm 105}$ & $123_{ \pm 170}$ & $301_{ \pm 80}$ & $85_{ \pm 141}$ & $77_{ \pm 205}$ \\
\hline$\left[20^{\circ}, 30^{\circ}\right]$ & $11_{ \pm 9}$ & $-37_{ \pm 44}$ & $99_{ \pm 3}$ & $273_{ \pm 157}$ & $-6_{ \pm 50}$ & $138_{ \pm 35}$ \\
\hline
\end{tabular}

\subsection{Discussion}

As is described in Monet et al. (1998), the USNO A catalogs are based on scans of 3 surveys at 4 bandpasses $(O, E, J$ and $R)$. They cover the entire sky to a limiting magnitude of about 20 depending of the wavelength. However, due to the calibration procedure, it is recommended to do local recalibration of the astrometry and photometry. See for further details (Monet et al. 1998). Many objects brighter than the 11th magnitude are replaced by GSC stars or Tycho stars (depending on the catalog versions) because of the saturation on the plates. The observations of optical counterparts of ICRF radio sources and the computations of their positions using USNO A2.0 stars appear to be a very efficient way to recalibrate this catalog locally. An example of the use of such calibration is given in Fienga et al. (2002). In that work, observations of the 9th satellite of Saturn, Phoebe, are presented. Positions of this satellite were deduced from a 2-years observations campaign reduced with USNO A2.0 stars. A significant offset of about 0.3 arcsec was detected in declination. Thanks to our observations of optical counterparts of ICRF sources and the results presented in this article, it was possible to identify this offset with a zonal error of the USNO A2.0 catalog and to correct it.

The GSC II and the extracted version catalog GSC 2.2 appears to be a good comparison tool (see GSC astrometric calibration $^{1}$ ). Theses catalogs are also based on scans of

\footnotetext{
${ }^{1}$ www-gsss.stsci.edu/gsc/gsc2/calibrations/ astrometry/
}

2 surveys at 3 bandpasses and 2 epochs. The limiting magnitude is 18.5 in $\mathrm{F}$ and 19.5 in J. Even if the external accuracies are equivalent to the USNO A uncertainties, the astrometric calibration provides a less corrupted data base for two reasons. First, differential refraction corrections are included. Second, systematic pattern maps are formed by averaged residuals found by comparison between computed positions and positions of ACT or Tycho reference stars. These maps, called astrometric masks, are used to correct the catalog from zonal and regional errors. By this method, the GSC II and the GSC 2.2 catalogs are corrected from a part of the systematic errors. However, the magnitude-dependent systematic errors could still remain.

As far as our study goes, the descriptions of the calibration methods used in the USNO and the GSC II catalogs fit quite closely the results we have found for the external accuracy of these two catalogs. It becomes obvious than the USNO A2.0 catalog needs regional or zonal corrections for stars fainter than the 11th magnitude. On the other hand, the preliminary version of the GSC II catalog seems to be garanteed against such regional or zonal distortions and to have a good external accuracy.

\section{Conclusion}

38 optical counterparts of ICRF radio sources were observed at the Observatoire de Haute Provence from December 3-10 2001. From these observations, positions were deduced 
Table 5. Means and standard deviations, in mas, of the Tycho- 2 minus USNO A2.0 and GSC $2.2 \alpha$ and $\delta$ of common stars in 8 observed fields. Besides the name of the ICRF sources around which the common stars were found, there appears the number of Tycho- 2 stars found respectively in the GSC2.2 and the USNO A2.0 catalogs. The differences were computed only when there are 4 or more common stars.

\begin{tabular}{lccrrrr}
\hline \hline $\begin{array}{l}\text { ICRF } \\
\text { Sources }\end{array}$ & GSC & UA2 & \multicolumn{2}{c}{ Tycho-GSC } & \multicolumn{2}{c}{ Tycho-UA2 } \\
& & & $\begin{array}{r}\Delta \alpha \cos \delta \\
{[\mathrm{mas}]}\end{array}$ & $\begin{array}{r}\Delta \delta \\
{[\mathrm{mas}]}\end{array}$ & $\begin{array}{r}\Delta \alpha \cos \delta \\
{[\mathrm{mas}]}\end{array}$ & $\Delta \delta$ \\
\hline $0219+428$ & 9 & 7 & $-54_{ \pm 146}$ & $106_{ \pm 119}$ & $-15_{ \pm 293}$ & $3_{ \pm 289}$ \\
$0309+411$ & 10 & 7 & $-53_{ \pm 153}$ & $70_{ \pm 52}$ & $-8_{ \pm 286}$ & $-134_{ \pm 229}$ \\
$0423+051$ & 5 & 5 & $10_{ \pm 165}$ & $188_{ \pm 105}$ & $-35_{ \pm 183}$ & $70_{ \pm 156}$ \\
$0518+165$ & 4 & 3 & $-259_{ \pm 313}$ & $138_{ \pm 256}$ & & \\
$0722+145$ & 8 & 6 & $-93_{ \pm 232}$ & $201_{ \pm 180}$ & $4_{ \pm 202}$ & $-18_{ \pm 157}$ \\
$0735+178$ & 5 & 4 & $-80_{ \pm 104}$ & $86_{ \pm 275}$ & $-352_{ \pm 158}$ & $-188_{ \pm 108}$ \\
$0754+100$ & 4 & 2 & $682_{ \pm 983}$ & $8_{ \pm 87}$ & & \\
$0839+187$ & 5 & 4 & $-166_{ \pm 216}$ & $269_{ \pm 274}$ & $16_{ \pm 215}$ & $-29_{ \pm 116}$ \\
\hline
\end{tabular}

using the USNO A2.0 catalog and the preliminary version of the GSC II catalog. Comparisons between the deduced positions of the optical counterparts and ICRF positions as well as with other published positions of the same objects were made. It appears than the USNO A2.0 catalog has a zonal error of $(0.254 \pm 0.153)$ arcsec in declination for stars with declinations between 0 and $+45 \mathrm{deg}$. The preliminary version of the GSC II catalogs does not have the same distortions. No significant offset is found in right ascension or declination. These results are in good agreement with the descriptions of the calibration methods used to set up the USNO A2.0 and the GSC II catalogs.

In the astronomical community, the USNO A2.0 catalog is widely used due to its high density of stars per square deg. Most of the common sky surveys use the USNO A2.0 catalog in order to calibrate their astrometry. However, many users reduce their fields without taking into account the warnings given by Monet et al. concerning possible local astrometric and photometric distortions. We would like to emphasize the need of such zonal or regional corrections at least if an accuracy better than 0.4 arcsec is needed.

By this work, we show that the observations and the study of the positions of optical counterparts of ICRF radio sources provide a powerful tool for the detection and removal of systematic errors in a stellar catalog as well as the estimation of its external accuracy. This new method is limited by the precision of the optical counterpart observations and by the density of available ICRF sources in the sky. We will continue the observations of such objects.

Acknowledgements. These observations have been made possible thanks to the CNRS (Centre National de la Recherche Scientifique), the Institut des sciences de l'Univers/CNES through the Programme National de Planétologie and the Institut de mécanique céleste/Bureau des Longitudes.

We also wish to thank the staff of the Observatoire de HauteProvence (OHP) for their efforts and their help during the campaign.

The Guide Star Catalog-II is a joint project of the Space Telescope Science Institute and the Osservatorio Astronomico di Torino. Space Telescope Science Institute is operated by the Association of Universities for Research in Astronomy, for the National Aeronautics and Space Administration under contract NASA5-26555. The participation of the Osservatorio Astronomico di Torino is supported by the Italian Council for Research in Astronomy. Additional support is provided by European Southern Observatory, Space Telescope European Coordinating Facility, the International GEMINI project and the European Space Agency Astrophysics Division.

Finally, we also thank warmly M. Schultheis, F. Sevre and C. Alard for their help.

\section{References}

Alard, C. 2002, private communication

Arias, E. F., Charlot, P., Feissel, M., \& Lestrade, J.-F. 1995, A\&A, 303, 604

Assafin, M., Andrei, A. H., Vieira Martins, R., et al. 2001, AJ, 552, 380

Banse, K., Grosbol, P. J., \& Baade, D. 1991, Astronomical Data Analysis Software and Systems I, PASP Conf. Ser., 25, 120

Charlot, P. 1990, AJ, 99, 1309

Da Silva Neto, D. N., Andrei, A. H., Vieira Martins, R., \& Assafin, M. 2000, AJ, 119, 1470

ECLIPSE $4.22002^{2}$

ESAB 1997, The Hipparcos and Tycho Catalogs (ESA SP-1200)

Fey, A. L., \& Charlot, P. 1997, ApJS, 111, 95

Feissel, M., \& Mignard, F. 1998, A\&A, 331, L33

Fienga, A. 1999, Ph.D. Thesis., Observatoire de Paris

Fienga, A., Arlot, J.-E., Baron, N., et al. 2002, A\&A, 391, 767

Fienga, A., Colas, F., Lecacheux, J., \& Berthier, J. 2002, A\&A, in preparation

IAU 1997, Proc. of the Twenty-Three General Assemblee (Kyoto, Japan)

Johnston, K. J., Fey, A. L., Zacharias, N., et al. 1995, AJ, 110, 880

Kovalevsky, J., Lindegren, L., Perryman, M. A. C., et al. 1997, A\&A, 323,620

Ma, C., Arias, E. F., Eubanks, T. M., et al. 1998, AJ, 116, 516

Monet, D., Bird A., Canzian, B., et al. 1998, The USNO-A2.0 Catalog (U.S. Naval Observatory, Washington DC)

Morrison, J. E., Roeser, S., McLean, B., Bucciarelli, B., \& Lasker, B. 1999, VizieR Online Data Catalog 1254

STScI $2001^{3}$

Urban, S. E., Corbin, T. E., \& Wycoff, G. L. 1998, AJ, 115, 216

Vieira Martins, R. 2001, private comunication

Zacharias, N., Zacharias, M. I., Hall, D. M., et al. 1999, AJ, 118, 2511

${ }^{2}$ wwW.eso.org/projects/aot/eclipse/
3 www-gsss.stsci.edu/gsc/gsc2/GSC2home.htm 